Rapport - Société canadienne d'histoire de l'Église catholique

\title{
En feuilletant les registres des Postes du Roi
}

\section{René Bélanger}

Volume 32, 1965

URI : https://id.erudit.org/iderudit/1007329ar

DOI : https://doi.org/10.7202/1007329ar

Aller au sommaire du numéro

Éditeur(s)

Les Éditions Historia Ecclesiæ Catholicæ Canadensis Inc.

ISSN

0318-6148 (imprimé)

1927-7075 (numérique)

Découvrir la revue

Citer cet article

Bélanger, R. (1965). En feuilletant les registres des Postes du Roi. Rapport -

Société canadienne d'histoire de l'Église catholique, 32, 37-41.

https://doi.org/10.7202/1007329ar

Tous droits réservés @ Les Éditions Historia Ecclesiæ Catholicæ Canadensis Inc., 1966
Ce document est protégé par la loi sur le droit d'auteur. L'utilisation des services d'Érudit (y compris la reproduction) est assujettie à sa politique d'utilisation que vous pouvez consulter en ligne.

https://apropos.erudit.org/fr/usagers/politique-dutilisation/ 


\section{En feuilletant les registres des Postes du Roi}

Le Concile de Trente impose aux pasteurs l'obligation de rédiger d'après une méthode consacrée, autant que possible en latin et dans des livres séparés, les actes de baptême, de confirmation, de mariage et de décès ainsi que l'état des âmes de leurs ouailles. L'enregistrement canonique des actes paroissiaux sera plus tard renforcé par le canon $\mathbf{4 7 0}$ de notre droit ecclésiastique.

Parallèlement à cette prescription, existait chez les supérieurs de la Compagnie de Jésus - chargée, de 1611 à 1782, du territoire qui nous occupe - l'usage de noter au jour le jour les événements marquants, tant comme aide-mémoire en vue du rapport annuel que pour établir une espèce de coutumier dans la maison ou la mission.

Assez souvent, celui qui tenait la plume sortait de la formule stéréotypée des actes pour narrer les circonstances des décès, etc., ou pour faire des réflexions de son cru. Ce qui leur donne du piquant et une forte saveur locale.

Les longs voyages en canot ou en raquettes obligèrent les missionnaires à réduire leur équipement au strict nécessaire et, par suite, à consigner dans un même cahier tout ce qu'ils avaient à enregistrer. Seulement, ils groupèrent par sections les baptêmes, les confirmations, les mariages, les sépultures, le journal et la liste des dons faits aux missions. Il en résulta une espèce de fouillis, ou encore " une forêt ", comme dit élégamment le père de la Brosse.

Il convient maintenant de décrire sommairement les livres en question. - Tadoussac, depuis longtemps la base des activités des premiers Européens au Canada, devenait à partir de 164l, sous l'étendard de la Sainte-Croix, la mission centrale du pays de Saguenay. Son premier régistre, qui reflétait sans doute le fruit des labeurs apostoliques des Pères De Quen et Albanel, a été perdu. Nous abordons donc notre étude avec le Second Régistre de Tadoussac: un cahier de $10^{\prime \prime} \times 7^{\prime \prime}$, sans couverture, contenant 62 feuillets. Le premier, le troisième et le quarante-troisième manquent. Au vingtième, on peut lire les noms de 182 Indiens confirmés par Mgr de Laval à Tadoussac en juin 1668, et à Québec le 5 juillet 1669. Ce précieux manuscrit est' à la garde du Séminaire de Québec.

A partir de 1691, on passe au Miscellaneorum Liber, au nom bien significatif : " petit in $-4^{\circ}$, de papier assez grossier, solidement relié et couvert en parchemin plus ou moins noirci par le temps ${ }^{1}$ ». Ce livre

1 Le Miscellaneorum Liber ou les Missions du Saguenay au $18^{\circ}$ s., abbé V.-A. Huard, Québec, 1912. 
de 180 pages, donné en 1686 par Dame Le Carlier, est le premier de la série des registres des "Postes du Roi ", conservés aux archives de l'Archevêché de Québec ${ }^{2}$.

Le suivant, d'un plus grand format, a toujours été appelé pour cette raison le Magnus Liber. Une étiquette à la première page de garde nous avertit que le cahier était en vente à Paris en 1713 chez Bélanger, qui y avait alors boutique, rue Dauphine, à l'enseigne de $A$ la petite vertu. Puis, à la deuxième page, l'inscription liminaire du père Coquart : "Commencé le $4^{\circ}$ de juin 1759 Daignent tous celıx qui écriront dessus se resouvenir dans leurs Saints Sacrifices et Prières de celui qui le commença. »

Au Magnus Liber, qui porte aux archives la cote " B ", succèdent quatre registres de format plus réduit : " $\mathrm{D}$ » reflète la période qui va de 1785 à 1796; " $\mathrm{E}$ », 1796-1810; " $F$ », 1815-1833; " $\mathrm{G}$ », 1834-1844. Ceux-ci ne contiennent que les actes de baptême, de mariage et de sépulture.

Dans ces pages jaunies par le temps, c'est tout un passé qui revit: audace tranquille des découvreurs et des explorateurs; vie aventureuse des coureurs de bois; vie souvent peu édifiante des engagés des postes; vie précaire des Indiens aux prises, chaque jour, avec les aléas de la chasse et la rigueur des éléments; vie ardente des missionnaires où alternent les courses apostoliques épuisantes et les pénibles hivernements à la mode indienne.

Ainsi, c'est en les feuilletant qu'on découvre le motif du choix de certaines appellations géographiques ou de quelques titulaires d'églises. Pointe-à-Michel, vis-à-vis de Betsiamits, perpétue le souvenir de Michel Mitshinakau et de son épouse, Angélique Kukumina, noyés accidentellement en cet endroit le 25 juin 1795 et inhumés ensemble dans le cimetière des Ilets-de-Jérémie. Sainte-Emérentienne du Grand Fond, desserte de La Malbaie, garde le souvenir d'Emérentienne Dufour, décédée en octobre 1747, fille de Joseph Dufour, maître de la ferme de La Malbaie, et de Marianne Tremblay.

De même, les surnoms indiens attirent bien vite l'attention du lecteur. On sait que jusqu'au début du $\mathrm{XIX}^{\mathbf{e}}$ siècle nos Indiens avaient, au lieu de ou en plus du nom de famille, un surnom qui décrivait une particularité de l'individu et reflétait la vie de ces enfants des bois. Plusieurs des plus anciens sont demeurés intraduisibles par suite de l'évolution de la langue montagnaise. Citons-en quelques-uns :

Pour une femme : fait semblant d'être jeune; celle qui s'est perdue; capable comme un homme; la pie.

Pour un homme : Paul, casseur d'ouragan; Pierre le borgne; la corne; le poilu; l'aigle; le castor; le cerf-volant; qui paraît toujours malade; bouillon de lièvre; a une main froide; qui en vaut deux.

2 Registre \& A v. 
Les actes de décès, pas toujours conventionnels, sont assez révélateurs. En voici quatre, rédigés par le père Coquart :

"Il est mort l'hyver dernier (1747-48) dans Manouan et dans Manicouagan environ 33 personnes tant hommes, femmes qu'enfants, de faim et de misère.

« Le 17 juin 1748 j'ay enterré à la porte du cimetière de Chekoutimi une enfant de 8 mois..., morte par le feu qui courait dans le bois. »

"Le 29 de novembre (1748), j'ai enterré à Chekoutimi Catherine, femme du païs de Manouan, aveugle depuis 30 ans, et qui n'avait pas veu de missionnaire depuis plus de 50.

"Pendant le même hyver (1752), on trouva la vieille Pierrot tenant entre ses bras son fils, tous deux gelés sur le Lac St-Jean.»

Messire Jean-Joseph Roy, prètre du Sëminaire de Quëbec et missionnaire de 1785 à 1795 , ne craignait pas, lui non plus, d'entrer dans les détails :

Inhumation de Jean-Marie Lamotte, natif de Saint-Augustin, âgé d'environ 23 ans, et de Louis Chauret, natif de Saint-Pierre-de-l'Ile, âgé d'environ 24, ans, respectivement commis et tonnelier du poste de Napissipi. * Etant partis du poste le 31 janvier 1787 pour aller à la chasse, furent trouvés gelés le lendemain près de la maison. "

- Jean-Baptiste Delage, décédé à Mingan le 17 juin 1787, âgé d'environ 63 ans, qui voyageait depuis plusieurs années dans les Postes du Domaine et de Mingan, et dont la femme est à St-Jean en l'Isle d'Orléans. »

Le 25 juillet 1791, à Portneuf, funérailles de a Marie-Madeleine... avec son enfant né après la mort de sa mère par une opération caesarienne. \$

Sépulture de « Jean-Baptiste Lyonnais, jeune homme natif de la ville de Québec, qui étant allé dans les Postes pour prendre l'air de l'eau salée, mourut à St-Joseph-des-Sept-Iles le cinq juin 1791, âgé d'environ 20 ans. '

En 1793, il bénit la fosse d'un jeune Indien de 2 ans, du nom de Christophe. "Son père étant ivre et ayant tiré un coup de fusil sur un français avec lequel il avait eu difficulté, le coup tua l'enfant.. Il est enterré aux Ilets dans le cimetière. Cet accident est arrivé en septembre 1792. Malheur aux effets de l'eau de vie!,

Bénédiction d'une autre fosse le 14 mai 1794, : François Labady, tonnelier de Portneuf, natif de Québec, âgé d'environ 24 ans, qui revenant de Québec en canot vira par un coup de vent et se noya dans la Baie appelée Mille-Vaches le 21 août dernier, et dont on a retrouvé, quelques jours après, une partie notable des ossemens sur le rivage, le reste ayant été mangé par les poissons. » 
Certaines sections des deux premiers registres, avons-nous dit, forment à proprement parler le Journal des Missions. A Tadoussac, à Chicoutimi, à Métabetchouan, le missionnaire imitait ce que ses confrères avaient fait à Québec quelques années auparavant en rédigeant le Journal des Jésuites. De son poste d'observation, il notait, parfois en français, parfois en latin, les menus faits comme les événements importants. Le tout dans une langue savoureuse, dans une latinité parfaite : ce sont des humanistes, formés dans les meilleurs collèges de France, qui écrivent. Sous la plume de ces anciens professeurs, le commis et l'anmurier ùu posie devienneni sespetivemeni : mercuiarce prceposiius et armorum faber; un capitaine de goélette : navis profectus; des canons : tormenta bellica; les Indiens se transforment en Montanenses, sylvicoloe, sylvestres.

A l'automne de 1690, l'annaliste, le père François de Crespieul, rapporte : le voyage du père Dalmas en compagnie de Pierre Lemoyne d'Iberville à destination du Port de Nelson, à la Baie d'Hudson; le siège de Québec par les Anglais Bostonnais; la pêche, par M. François de la Chevrotière, de 1500 poissons "dans l'Anse vers Manitounak * (aujourd'hui l'Ile-aux-Couleuvres, en face de Roberval).

A la date de 1742 , le père Jean-Baptiste Maurice consigne ce qui suit : "Dans le ler voyage de la goélette Mr (Jean-Baptiste) Gosselin, prêtre et chanoine de la Cathédrale de Québec, s'embarqua avec $\mathrm{Mr}$ Cugnet (François-Etienne, régisseur des Postes du Domaine du Roi) pour voir si dans les terres du Domaine il ne trouverait pas quelques plantes particulières. Il a eu le bonheur, dit-on, d'en rencontrer quelques-unes qui ont été estimées et reçeues au Jardin du Roy en France. »

Voici maintenant, du père Laure, un amusant fait divers : "Dans cet endroit-là (aux Ilets-de-Jérémie), le 23e de juillet 1735, au sortir de la prière du soir et de l'exhortation, vint s'échouer une baleine dans l'Anse au nord-ouest de la pointe. J'en avertis aussitôt les Français. Après avoir bien examiné avec mes lunettes d'approche, nous allâmes la reconnaître plus sûrement. De peur de quelque coup de vent qui l'emportât au large, on la mouilla le soir en l'amarrant avec un cable, et le matin on la remorqua à la pointe du gros Ilet, à la croix, où je lui trouvai 60 pieds de longueur, 30 pieds de hauteur et 30 à peu près de diamètre. J'entrai dans la large embouchure de sa gueule, mais je n'osai avancer au-delà du gosier, de peur que, quelque funeste ressort venant à se resserrer, je ne devinsse pis que Jonas. Elle a produit 93 barriques d'huile. Les deux barbes qui sont à la chapelle de Chekoutimi, et que j'ai apportées à mon retour, doivent servir de preuve que la prière ne tue pas les sauvages. "

Enfin, les registres sont émaillés ici et là d'originalités. Cinq pages du Miscellaneorum Liber ont été rédigées par le père Gélase Delestage, récollet, qui vint donner la mission à Tadoussac et à Chicoutimi en 1716. Devant le laconisme extrême de ces actes, parsemés au surplus de nombreuses fautes de grammaire et d'orthographe, les Jésuites n'ont pu réprimer un sourire, accentué peut-être par le fait que ce brave Père 
ótait un Canadien de naissance et un frère mineur. Dans la moitié de la page restée en blanc, le père Laure écrit d'abord ce qui suit :

\section{Diu sub judice lis fuerit \\ Siste, viator!}

Plus tard, le père de la Brosse ajoute, en guise de bouquet, le quatrain que voici :

Vere prius flores, astu numerabis aristas,

Poma per autumnum, frigoribusque nives;

Quam voces perperam exaratas ab illo Rev. Patre Gelasio de

Lestage contra omnes tum Grammatica, tum Orthographice leges.

Le père Coquart ne manque pas, lui non plus, d'originalité à ses heures. En 1758, à Chicoutimi, il officie au mariage de "Marguerite, fille de la vieille Monique, veuve de je ne scay qui aux 3 Rivières. » Trois ans plus tard, au même endroit, il baptise l'enfant de cette Marguerite : "Parein Mr Moreau, la mareine Jeanne, $\mathrm{f}(\mathrm{emme})$ de François qui ont nommé l'enfant Je n'en say rien; mais vous auriez $d u$ le savoir!»

En voilà assez, sans doute, pour souligner l'importance et l'intérêt de ces documents, source de l'histoire d'un immense pays, exclusivement réservé pendant deux siècles à la traite et aux missions.

(Mgr) René BÉLANGer, p.d. 